\title{
AN ACCELERATOR RESONANTLY COUPLED WITH AN ENERGY STORAGE (ARES) FOR THE KEKB
}

\author{
Y. Yamazaki, K. Akai, N. Akasaka, E. Ezura, T. Kageyama, F. Naito, T. Shintake, and Y. Takeuchi, \\ KEK, Natinal Laboratory for High Energy Physics, Oho 1-1, Tsukuba-shi, Ibaraki-ken 305, Japan
}

In a large ring with extremely heavy beam loading, such as a B-factory, it is possible that the accelerating mode, itself, gives rise to a longitudinal coupled-bunch instability. In order to solve this problem, T. Shintake has proposed to attach a TE015-mode storage cavity to an accelerating cavity. It has subsequently been shown that the system can be put into practical use if a coupling cavity is added in between the two cavities. The three-cavity system, which is now refered to as an accelerator resonantly coupled with an energy storage (ARES), is under develpment for the KEKB.

\section{INTRODUCTION}

At this conference the development of the KEKB roomtemperature (normal-conducting) RF system is being systematically reported in detail. The newly invented accelerating cavity [1-3] will be used for this system. This cavity, refered to as "an accelerator resonantly coupled with an energy storage (ARES)," is actually a coupled-cavity system comprising three cavities: an acclerating cavity (acavity) and an energy-storage cavity (s-cavity) with a coupling cavity (c-cavity) in between (see Fig. 1). This report introduces the concept of ARES by presenting the reason why it is necessary.

It has been empirically confirmed by measuring the RF characteristics of ARES cold models (see Ref. [4]) that ARES behaves as theoretically expected in this report. The total consistent set of RF parameters for the KEKB is presented in Ref. [5], showing that ARES can meet the requirements for the KEKB.

The accelerating cavity of the ARES should be a "damped cavity," the higher-order modes of which are sufficiently damped in order to suppress any coupled-bunch instability. Among the various proposed damped cavities, the choke-mode cavity, which was independently devised by $\mathrm{T}$. Shintake [6] and K. Akai [7], has been chosen [8, 9] for the KEKB. The result of the first high-power test of the chokemode cavity is presented in Ref. [10]. Since the performances of the ceramics parts are critical technical issues for this kind of high-power RF system, the performance of the higher-order mode absorbers made of $\mathrm{SiC}$ is reported in Ref. [11], while the design of the input coupler is described in Ref. [12].

\section{THE COUPLED-BUNCH INSTABILITY ARISING FROM THE ACCELETATING MODE}

In order to store a high-beam current in an electron/positron storage ring, the threshold current of any coupled-bunch instability must be sufficinently high. For this purpose it is essential to sufficiently damp any dangerous higher-order modes in the accelerating cavities. This, however, is not sufficient for a large ring with extremely heavy beam loading, such as a B-factory. The accelerating mode, itself, may give rise to a longitudinal (synchrotoron) coupled-bunch instability for the following reason [13].

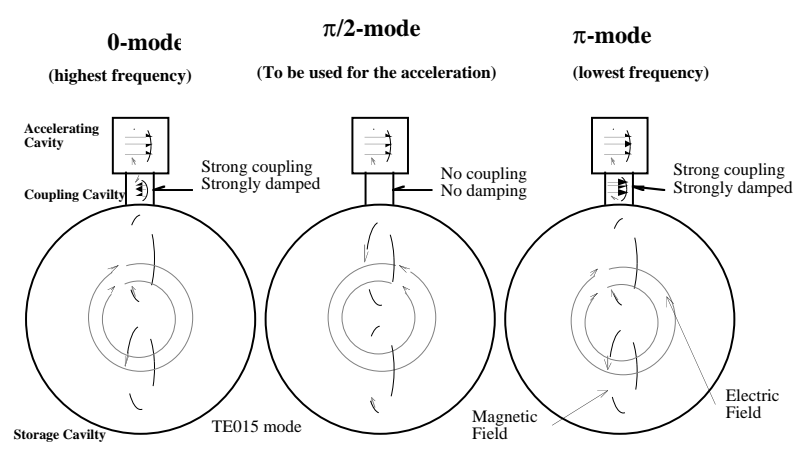

Figure 1: Three-cavity system with a coupling cavity in between the accelerating and storage cavities.

In order to keep the quantum lifetime sufficiently long, the bunch of the beam is accelerated out of phase from the crest of a sinusoidal function of the RF-field oscillation (this phase difference is refered to as a synchronous phase $\left(\phi_{\mathrm{S}}\right)$ ). As a result, the beam induces a reactive component in an accelerating cavity. In order to compensate for the reactive component, the cavity is detuned by an amount of $\Delta \mathrm{f}$, given by [14]

$$
\frac{2 \Delta \mathrm{f}}{\mathrm{f}} \cong \frac{\mathrm{R}_{\mathrm{Sh}}}{\mathrm{QL}} \frac{\mathrm{I}}{\mathrm{V}_{\mathrm{C}}} \sin \phi_{\mathrm{s}}
$$

where $\mathrm{R}_{\mathrm{Sh}}$ and $\mathrm{Q}_{\mathrm{L}}$ are the shunt impedance and the loaded $\mathrm{Q}$-value of the accelerating mode, respectively. The average beam current $(\mathrm{I})$ is accelerated by the cavity voltage $\left(\mathrm{V}_{\mathrm{C}}\right)$.

Usually, the detuning contributes to the damping of the $\mu=0$ mode of longitudinal coupled-bunch oscillations (Robinson damping), where $\mu$ designates the mode number in such a way that the phase difference between one bunch and the following one is given by $2 \pi \mu / \mathrm{N}_{\mathrm{b}}\left(\mathrm{N}_{\mathrm{b}}\right.$ is the number of bunches in a ring). When the beam is executing the longitudinal coupled-bunch oscillation of the $\mu$ mode, it has a frequency component given by

$$
\mathrm{f}^{ \pm} \mu \mathrm{n}=\mathrm{nN}_{\mathrm{b}} \mathrm{f}_{\mathrm{r}} \pm\left(\mu \mathrm{f}_{\mathrm{r}}+\mathrm{f}_{\mathrm{s}}\right)
$$


where $f_{s}$ is the synchrotron oscillation frequency. If the $f^{-} \mu, n$ component of the impedance of a ring is higher than the $\mathrm{f}^{+} \mu, \mathrm{n}$ component, the oscillation is damped, and vice versa. Since the $\mathrm{f}^{-} \mu=0, \mathrm{n}$ component of the impedance is increased by the detuning, while the $\mathrm{f}^{+} \mu=0, \mathrm{n}$ component is decreased, the $\mu=0$ mode oscillation is damped.

However, if the beam current is extremely high, the detuning frequency becomes very high, and if, in addition, the circumference of the ring is very large, that is, the revolution frequency is low, we must detune the cavity by two or three times as much as the revolution frequency. During the course of storing the beam the accelerating-mode frequency passes through $f-f_{r}, f-2 f_{r}$ (see Fig. 2) and so forth, resulting in extremely high growth rates of the $\mu=h-$ $1, \mathrm{~h}-2$ modes, respectively.

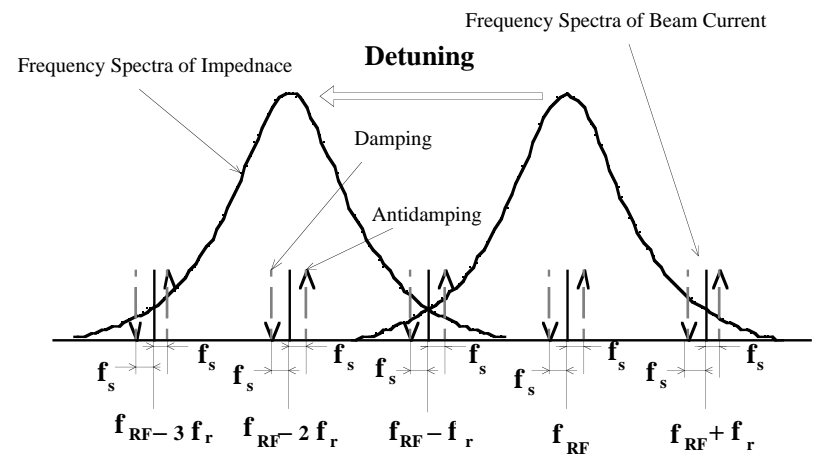

Figure 2: Frequency spectrum of the cavity impedance and beam current. The RF frequency is designatned by $f_{R F}$.

\section{ARES}

One may argue that the instability can be avoided by giving up the detuning. Although this is theoretically correct, it is practically unrealistic, since an extremely high power must be provided to the cavity in order to maintain the necessary cavity voltage against the high reflected power arising from the large reactive component induced by the beam.

Another way is to decrease the detuning. In order to gain a deeper insight into the detuning, it may be useful to rewrite Eq. (1) as

$$
2 \Delta \mathrm{f}=\frac{\mathrm{P}_{\mathrm{b}} \tan \phi_{\mathrm{S}}}{2 \pi \mathrm{U}} .
$$

This is nothing but the beam loading $\left(\mathrm{P}_{\mathrm{b}}\right)$ divided by the stored energy (multiplied by the tangent of $\phi_{\mathrm{S}}$ ). It should be noted that $\phi_{\mathrm{S}}\left(\mathrm{P}_{\mathrm{b}}\right.$ either) is not an adjustable parameter in a $\mathrm{B}$ factory, since the cavity voltage is determined by sufficiently decreasing the bunch length in order to realize an extremely high luminosity (the bunch length should be shorter than the extremely small beta function $\left(\beta^{*}\right)$ at the colliding point).
Then, we can just increase the stored energy;however, it is necessary to increase it by an order of magnitude. Usually, the high power dissipation $(\mathrm{Pc})$ is induced by increasing the stored energy, since the ratio of the power dissipation to the stored energy is fixed as the inverse of a quality factor multiplied by an angular frequency $\left(\mathrm{P}_{\mathrm{C}}=\omega \mathrm{U} / \mathrm{Q}_{0}\right.$, where $\mathrm{Q}_{0}$ is an intrinsic Q-value). In order to maintain the power dissipation within the practically tolerable limit, although the use of a superconducting cavity is one solution, we cannot increase $\mathrm{U}$ as much as we want, since $\mathrm{U}$ is related to an unadjustable parameter $\left(\mathrm{V}_{\mathrm{C}}\right)$.

It was proposed by Shintake [1] to attach an s-cavity operating in a very high-Q TE015 mode to an a-cavity. Such an s-cavity has been successfully used in a SLED system. In order to realize his concept, it is necessary to attach one more cavity, refered to as a coupling cavity (c-cavity), in between the a- and s-cavities, as schematically shown in Fig. 1. The thus-formed three-cavity system is now refered to as ARES.

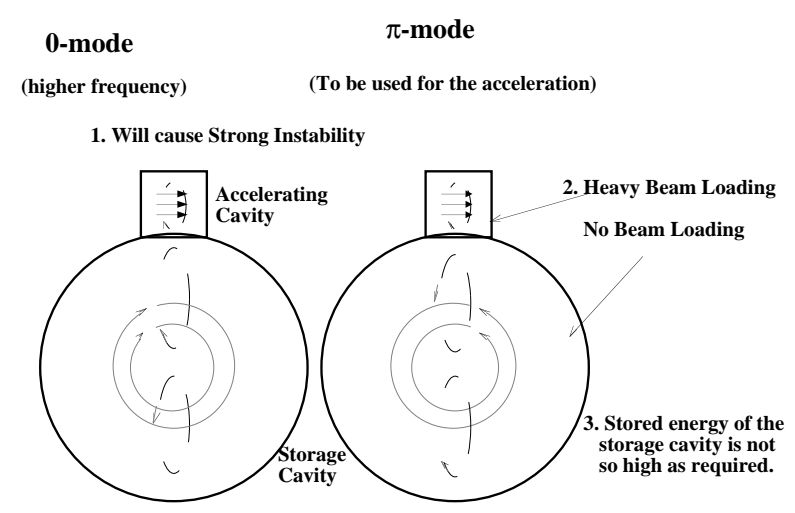

Figure 3: Two-cavity system comprising an accelerating cavity and a storage cavity.

The c-cavity is necessary for the following reason. Suppose that one immediately attaches the s-cavity to the acavity as shown in Fig. 3. If one mode is used as an accelerating mode, the other mode as a parasitic mode gives rise to a very strong beam instability. Second, these modes, refered to as the $\pi$-mode and the 0 -mode, have no group velocity. Since the a-cavity is heavily beam-loaded, while the s-cavity is not, the stored-energy distribution is significantly unstable against the beam loading. Finally, if the resonant frequencies of two cavities are identical, as is usually the case in a coupled-cavity system, the stored energy of the s-cavity becomes the same as that of the acavity. In order to increase the stored energy of the s-cavity by an order of magnitude, one must significantly deviate the frequency of the a-cavity from that of the s-cavity. Then, the energy flow from the s-cavity to the a-cavity would be seriously blocked, resulting in a practically isolated cavity system, rather than a closely coupled system. 
These three problems can be solved by attaching the ccavity and operating the three-cavity sytem in the $\pi / 2$ mode. The energy flow between the a-cavity and s-cavity is significantly improved by the famous characteristic of the $\pi / 2$ mode operation; that is, the system is stable against the beam loading. The last problem is also solved by adjusting the coupling constant $\left(\mathrm{k}_{\mathrm{a}}\right)$ between the a-cavity and c-cavity with respect to the coupling $\left(\mathrm{k}_{\mathrm{S}}\right)$ between the s-cavity and ccavity, since the ratio of the stored energy $\left(\mathrm{U}_{\mathrm{S}}\right)$ of the scavity to that $\left(\mathrm{U}_{\mathrm{a}}\right)$ of the a-cavity is given by $[15,16]$

$$
\left(\mathrm{U}_{\mathrm{S}} / \mathrm{U}_{\mathrm{a}}\right)=\left(\mathrm{k}_{\mathrm{a}} / \mathrm{k}_{\mathrm{S}}\right)^{2} .
$$

It may sound paradoxical that we can solve the first problem, (the strong beam instability arising from the parasitic mode) by adding one more cavity (by increasing the number of parasitic modes). Fortunately, no field is excited in the ccavity operating in the $\pi / 2$ mode while very high fields exist in both the 0 -mode and $\pi$-mode (see Fig. 1). Therefore, if one installs a coupler to extract power from the c-cavity, it is effective on the 0 -mode and $\pi$-mode, while having no effect on the accelerating $\pi / 2$ mode. This was confirmed by a lowpower test of a model at one-fifth scale, as can be seen in Ref. [4].

Furthermore, the frequencies of the 0 -mode and $\pi$-mode are approximately symmetrically distributed with respect to the $\pi / 2$ mode. Thus, the antidamping effect of the mode $\mu$ beam oscillation due to the $\mathrm{f}^{+} \mu, \mathrm{n}$ component of the impedance of one of the two parasitic modes is approximately canceled by the damping effect of the $\mathrm{f}^{-} \mu, \mathrm{n}$ component arising from the other parasitic mode.

\section{CONCLUSIONS}

The discussion given here was based upon two assumptions. First, the system is made of perfect conductors. Second, the storage cavity has only one mode, or, practically speaking, the TE015 mode is well separated from the other modes. Since neither assumption is correct, we have carefully studied these two effects, using the coupledresonator model calculation for the first problem [2] and a three-dimensional analysis for the second one [3]. The result was quite promising, although an extensive study was necessary in order to sufficiently separate the TE015 mode from the other modes.

As long as no serious problem is found during the course of development, ARES will be fully used for the KEKB. The RF-related parameters of both the low-energy ring (LER) and high-energy ring (HER) are presented in Ref. [5].

Finally, it should be emphasized that ARES will reveal the full advantage of its extremely high stored energy when missing-bunch (or partailly filled bunch) operation [17] is necessary in order to suppress any ion-trapping effects. The thus-introduced phase modulation will be minimized by increasing the stored energy.

\section{REFERENCES}

[1] T. Shintake, Part. Accel. 44, 131 (1993).

[2] Y. Yamazaki and T. Kageyama, Part. Accel. 44 , 107 (1994).

[3] K. Akai and Y. Yamazaki, Part. Accel. 46 , 197 (1994).

[4] "RF Charcteristics of ARES Cold Models," N. Akasaka, K. Akai, T. Kageyama, T. Shintake, and Y. Yamazaki,, WPQ09 in this Conference.

[5] "Design of the KEKB RF System", K. Akai, E. Ezura, and Y. Yamazaki, WPQ08 in this Conference.

[6] T. Shintake, Jpn. J. Appl. Phys. Lett. 31 , L1567 (1992).

[7] K. Akai, J. Kirchgessner, D. Moffat, H. Padamsee, J. Sears, T. Stowe and M. Tigner, Proc. 15th Int. Conf. on High Energy Accelerators, 757 (1992).

[8] T. Kageyama, N. Akasaka, Y. Takeuchi, and Y. Yamazaki, Proc. 4th European Par. Accel. Conf. , 2098 (1994).

[9] N. Akasaka, T. Kageyama, and Y. Yamazaki, Proc. 4th European Par. Accel. Conf., 2137 (1994).

[10] "Development of a HOM-Damped Cavity for the KEK B-Factory(KEKB)," T. Kageyama, K. Akai, N. Akasaka, E. Ezura, F. Naito, T. Shintake, Y. Takeuchi, and Y. Yamazaki, WPQ17 in this Conference.

[11] "HOM Absorber for the KEKB Normal Conducting Cavity," Y. Takeuchi, K. Akai, N. Akasaka, E. Ezura, T. Kageyama, F. Naito, T. Shintake, and Y. Yamazaki , WPR05 in this Conference.

[12] "Input Coupler for the KEKB Normal Conducting Cavity," F. Naito, K. Akai, N. Akasaka, E. Ezura, T. Kageyama, T. Shintake, Y. Takeuchi, and Y. Yamazaki, WPR09 in this Conference.

[13] An Asymmetric B Factory based on PEP Conceptual Design Report (1991).

[14] P. B. Wilson, SLAC-PUB-2884 (1982).

[15] E. A. Knapp, B. C. Knapp, and J. M. Potter, Rev. Sci. Instr. 39, 979 (1968)

[16] Y. Morozumi, T. Kageyama, and Y. Yamazaki, Proc. 1990 Linear Accel. Conf., 153 (1990).

[17] Y. Yamazaki, M. Kihara, and H. Kobayakawa, KEK Report 83-17 (1983). 